\title{
Effects of feedback on discriminative avoidance acquisition and extinction in the gerbil (Meriones unguiculatus)
}

\author{
PETER F. GALVANI \\ State University-of New York, College at Brockport, Brockport, New York 14420
}

\begin{abstract}
An exteroceptive feedback stimulus (FS) consequent upon an avoidance response was found functionally equivalent to the traditional warning signal termination (WST) contingency in reinforcing shuttlebox avoidance in the Mongolian gerbil. Thus, presentation of an FS in acquisition led to successful avoidance learning even in the absence of immediate WST, while no evidence of summation of the FS and WST contingencies was obtained. In extinction, relatively abrupt loss of responding was observed regardless of the programmed consequences of an avoidance response. These results were discussed in terms of an informational hypothesis of avoidance behavior, especially with respect to the notion of redundancy of information.
\end{abstract}

One recent challenge to the traditional two-factor theory (Mowrer, 1947) interpretation of discriminative avoidance learning has been based on the significance of response-produced feedback in avoidance learning. Such formulations (e.g., Bolles \& Grossen, 1969) have stressed that the traditional warning signal termination (WST) contingency, emphasized within two-factor theory as providing reinforcement for the avoidance response via fear reduction, might be better conceptualized as providing information to the subject. When a subject performs the instrumental avoidance act, several responsecontingent stimulus changes, exteroceptive and interoceptive, may serve as sources of feedback (e.g., WST) and proprioceptive feedback from the response. Any one of these response-contingent stimulus changes might be viewed as signaling successful shock avoidance, and it is the informational value of such stimulus changes that is functionally significant in the acquisition and maintenance of the avoidance response. Studies of the effects of exteroceptive feedback stimuli (FS) on avoidance acquisition have lent support to this cognitive, informational hypothesis by demonstrating that presentation of an FS consequent upon the avoidance response leads to successful avoidance learning, even in the absence of the WST contingency (Bower, Starr, \& Lazarovitz, 1965; D’Amato, Fazzaro, \& Etkin, 1968; Keehn \& Nakkash, 1959). Such data suggest that the most important function of the WST contingency is that it provides information to the subject about the consequences of responding.

As Katzev and Hendersen (1971) have demonstrated,

The present research was supported by National Science Foundation Grant B043556-001, awarded to the author. The author thanks Michael Twitty for comments on an earlier version of the manuscript. Reprints may be obtained from the author, Department of Psychology, State University College at Brockport, Brockport, New York 14420. however, the efficacy of a response-contingent FS does not appear to be independent of the WST contingency. These authors found that if immediate WST was programmed in acquisition, then the exteroceptive FS failed to control behavior. They reasoned that when both FS and WST are response contingent, the FS is a redundant source of information; its presentation provides no information beyond that provided by termination of the WS. These authors showed, however, that if WST was delayed following an avoidance response, while the FS occurred immediately, then the FS acquired control over avoidance.

The major purpose of the present experiment was to provide a systematic replication of several of the conditions in Katzev and Hendersen's (1971) Experiments 1 and 2 , combined in one design which permitted the assessment of the efficacy of feedback when redundant as well as nonredundant in both acquisition and extinction. Several important modifications of Katzev and Hendersen's procedures were employed. The present study used a longer, 90-sec intertrial interval (ITI), since recent evidence has shown (Morris, 1974) that the shorter (mean $35 \mathrm{sec}$ ) ITI employed by Katzev and Hendersen may have decreased the effectiveness of the FS, thus making feedback effective only when nonredundant. Also, the FS was made equivalent in length to WST delay in the appropriate groups. In Experiment 1 of Katzev and Hendersen, FS duration was less than WST delay, conditions shown by Bolles and Grossen (1969, Experiment 3) to make feedback ineffective. This might explain the failure of feedback to maintain avoidance, as opposed to its redundancy, in Katzev and Hendersen's study. Finally, the present experiment employed Mongolian gerbils as subjects. To the best of the author's knowledge, the efficacy of the exteroceptive feedback contingency has never been evaluated in the gerbil, and the present study attempted to determine 
whether the effects of feedback may be generalized to this relatively new laboratory species.

\section{METHOD}

\section{Subjects}

The subjects were 60 naive adult male gerbils obtained from Tumblebrook Farms, Inc., West Brookfield, Massachusetts. All animals were group housed in a continuously illuminated room and maintained on ad-lib food and water.

\section{Apparatus}

Two identical toggle-fluor shuttleboxes $(13.5 \times 13 \times 40 \mathrm{~cm})$ made by Scientific Prototype (Model A100S) were employed (see Galvani, 1971, for more detailed description). Each shuttlebox was enclosed in a ventilated sound-attenuated chamber illuminated by a 7.5-W incandescent lamp. The tonal WS $(2,500 \mathrm{~Hz})$ employed in the experiment was delivered through an 8-cm speaker, centered above the shuttlebox, by a BRSForinger AU-902 audio generator. The WS raised the ambient sound level ( $82 \mathrm{~dB}$ with ventilating fan on) about $3 \mathrm{~dB}$ on the $C$ scale of a General Radio Type 1561-A sound-level meter. The FS consisted of the co-extensive offset of the 7.5-W light and the ac ventilating fan (which produced a background masking noise). The US was a $.5-\mathrm{mA}$ (nominal) .5 -sec scrambled electric shock delivered to the shuttlebox grid floor by a Grason-Stadler Model GS700 shock generator. Electromechanical programming and data recording equipment were located next to the soundattenuated chambers.

\section{Procedure}

After a 10-min adaptation period in the shuttlebox, each gerbil received a single 100-trial acquisition session, followed immediately by a 100-trial extinction session, with all trials presented at a $90-\mathrm{sec}$ ITI. The WS-US interval was $5 \mathrm{sec}$ on acquisition trials, with shock onset occurring simultaneously with WS offset. Any shuttle response that occurred within $5 \mathrm{sec}$ of WS onset, in either acquisition or extinction, was defined as a CR. All CRs effectively precluded shock in acquisition. No shocks were delivered in extinction. If an animal failed to respond during the 5 -sec WS in extinction, the WS automatically terminated $5 \mathrm{sec}$ after its onset.

The design employed a total of five groups, 12 gerbils per group. Each treatment was labeled in terms of both the WST contingency-immediate (I) or delayed $10 \mathrm{sec}$ (D)-and whether or not a 10-sec FS occurred-feedback (F) or no feedback (N). The group labels indicate the acquisition-xtinction conditions, respectively: IN-IN, IF-IN, DF-DF, DF-DN, and DN-DN.

\section{RESULTS}

The mean number of CRs for each group is plotted in Figure 1 over 20-trial blocks in acquisition and extinction. All five groups are plotted separately in the upper panel of Figure 1, although for purposes of analysis Groups DF-DF and DF-DN were combined in acquisition. An analysis of variance was performed on the acquisition data of Figure 1, with WST (I vs. D) and feedback ( $\mathrm{F}$ vs. $\mathrm{N}$ ) as between-subjects variables, and trial blocks as a within-subjects variable. All three main effects were significant: WST $[F(1,56)=16.36$, $\mathrm{p}<.001]$, feedback $[\mathrm{F}(1,56)=4.25, \mathrm{p}<.05]$, and trial blocks $[\mathrm{F}(4,224)=49.02, \mathrm{p}<.001]$. The only reliable interaction was WST by Feedback $[\mathrm{F}(1,56)=$ $5.08, \mathrm{p}<.05]$. From Figure 1 it appears that the interaction between the WST and feedback contingencies
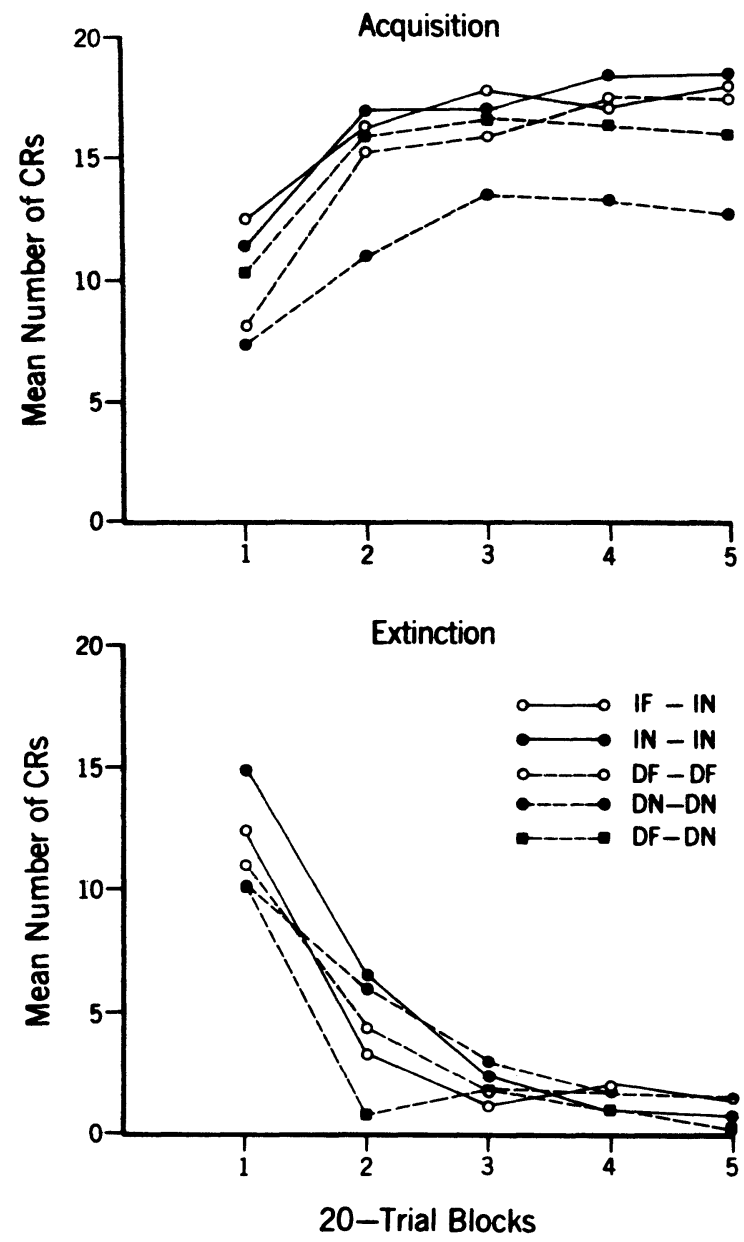

Figure 1. Mean number of avoidance CRs in 20-trial blocks of acquisiton (upper panel) and extinction (lower panel) as a function of whether the CR produced immediate (I) or delayed (D) WST and whether or not (F or N) it produced feedback. Each group is labeled according to acquisitionextinction treatment.

is due to the fact that feedback had no facilitative effect on avoidance learning when WST was immediate, but did enhance performance if termination of the WS was delayed. Indeed, follow-up $t$ tests showed that Group DN was reliably inferior to the other three acquisition treatments in terms of both total CRs (all ps $<.01$ ), and mean number of CRs on the final, 20-trial block of acquisition (all ps <.02). No other pairwise comparison was reliable for either $C R$ measure.

These acquisition data are straightforward. Gerbils which had some immediate, programmed consequence of a CR, whether FS, WST, or both, performed comparably, and were superior to those animals lacking either of these CR-produced stimulus changes.

The analysis of the extinction data involved specific tests of the redundancy notion. Presumably, following from Katzev and Hendersen (1971), one would expect that loss of redundant feedback would not affect extinction, whereas loss of nonredundant feedback 
(i.e., FS combined with delayed WST in acquisition) should result in abrupt extinction. As evident in the lower panel of Figure 1, persistence of the avoidance response was not great under any condition. Thus, all extinction comparisons included only Trial Blocks 1-3. Three extinction comparisons were selected a priori to evaluate the effects of loss of redundant (i.e., IF-IN vs. IN-IN) as opposed to nonredundant (i.e., DF-DN vs. DF-DF and DF-DN vs. DN-DN) feedback on resistance to extinction.

For all three comparisons, the trial blocks effect was reliable $[\mathrm{Fs}(2,44) \geqslant 40.3$, ps $<.001]$. The loss of redundant feedback had no effect on extinction, that is, IF-IN vs. IN-IN $[F(1,22)=2.88, p>.05]$, and the Groups by Trial Blocks interaction $[F(2,44)<1]$. The tests involving loss of nonredundant feedback were equivocal. In one case, the effect was nonsignificant: Groups DF-DN vs. DF-DF $[F(1,22)=2.16, p>.05]$ and the Group by Trial Blocks interaction $[F(2,44)=1.73$, $p>.05]$. On the other hand, while the Group DF-DN vs. DN-DN effect was not reliable $[F(1,22)=2.11$, $\mathrm{p}>.05]$, the Group by Trial Blocks interaction was significant in this comparison $[F(2,44)=3.97, p<.05]$. This interaction resulted from a more precipitous decrement in responding in Group DF.DN from Block 1 to Block 2 of extinction, relative to the response decrement of Group DN-DN. Presumably, this interaction indicates that loss of nonredundant feedback can facilitate extinction. With respect to comparable extinction comparisons reported by Katzev and Hendersen (1971, Experiment 2), the present extinction data are in agreement in that Group DF-DN extinguished more rapidly than Group DN-DN, but the failure of Group DF-DN to differ from DF-DF is clearly inconsistent with Katzev and Hendersen's results.

\section{DISCUSSION}

The present acquisition data point to the conclusion that presentation of an exteroceptive FS, following an avoidance response, leads to successful learning even in the absence of immediate WST. These results, obtained with gerbils in shuttle avoidance, are consistent with those reported for rats in both shuttle- and running-wheel avoidance (Bolles \& Grossen, 1969), as well as in barpress avoidance (D'Amato et al., 1968). The present results, on the other hand, do not demonstrate a summative effect of the feedback and WST contingencies as reported by Bolles and Grossen (1969, Experiment 3), and in this regard are in agreement with Katzev and Hendersen's (1971, Experiment 1) results. This pattern of acquisition results is consistent with the assertion by Bolles and Grossen (1969) that the WST and extrinsic feedback contingencies are functionally equivalent in establishing the avoidance response.

One major discrepancy with respect to the acquisition data of Katzev and Hendersen (1971, Experiment 2), however, is the present finding that Group DN was inferior to Group DF. Katzev and Hendersen reported that DF and DN treatments did not result in reliable differences in a trials-to-criterion measure. This comparison is misleading, however, in that these authors discarded 7 of 15 subjects in the DN treatment for failure to reach criterion, whereas there were no such discards from the DF group. Thus, these authors doubtless introduced a sampling bias such that those rats left in Group DN were better avoidance learners than rats in other groups in the design.

The extinction results showed that loss of redundant feedback (i.e., in Group IF-IN) did not accelerate rate of extinction relative to animals that never had an FS (IN-IN). Therefore, the present results imply that Katzev and Hendersen's data on the effects of loss of a redundant FS were not specific to Fischer ${ }_{344}$ rats, or the short ITI they employed, or the brief FS used in extinction. The results pertinent to loss of nonredundant feedback, however, were quite unexpected. While Katzev and Hendersen's data clearly showed nonredundant feedback was effective in response maintenance, such a result was reliably obtained in the present study only in the case of the DF-DN vs. DN-DN comparison; more rapid extinction was not found in Group DF-DN relative to DF-DF. The reasons for the inconsistency in these extinction comparisons are not clear, especially in light of the fact that the two baseline groups, DF-DF and DN-DN, were statistically equivalent in resistance to extinction $(F<1)$. It must be emphasized that persistence was not great under any condition, however, and this factor might have mitigated against obtaining reliable evidence for the efficacy of feedback.

In sum, the present acquisition results are consistent with a redundancy view of the functional significance of exteroceptive feedback in that an FS only facilitates performance in the absence of WST. The extinction results, however, provide only marginal support for the redundancy view, but loss of responding was abrupt under all conditions, perhaps rendering the extinction tests relatively insensitive.

\section{REFERENCES}

Bolles, R. C., \& Grossen, N. E. Effects of an informational stimulus on the acquisition of avoidance behavior in rats. Journal of Comparative and Physiological Psychology, 1969, 68, 90-99.

Bower, G., Starr, R., \& Lazarovitz, L. Amount of response-produced change in the CS and avoidance learning. Journal of Comparative and Physiological Psychology, 1965, 59, 13-17.

D'Amato, M. R., Fazzaro, J., \& Etkin, M. Anticipatory responding and avoidance discrimination as factors in avoidance learning. Journal of Experimental Psychology, 1968, 77, 41-47.

Galvani, P. F. The effects of partial reinforcement on the acquisition and extinction of avoidance behavior in gerbils. Psychonomic Science, 1971, 24, 242-244.

Katzev, R. D., \& Hendersen, R. W. Effects of exteroceptive feedback stimuli on extinguishing avoidance responses in Fischer344 rats. Journal of Comparative and Physiological Psychology, 1971, 74, 66-74.

KeEHN, D. J., \& NAKKaSh, S. Effect of a signal contingent upon an avoidance response. Nature (London), 1959, 184, 566-568.

Morris, R. G. M. Pavlovian conditioned inhibition of fear during shuttlebox avoidance behavior. Learning and Motivation, 1974, 5, 424-447.

Mowrer, O. H. On the dual nature of learning-a reinterpretation of "conditioning" and "problem solving." Harvard Educational Review, 1947, 17, 102-148.

(Received for publication June 12, 1977.) 